\title{
BURNOUT Y SATISFACCIÓN LABORAL: HALLAZGOS DIFERENCIALES POR GÉNERO
}

\section{BURNOUT AND WORK SATISFACTION: DIFFERENTIAL FINDINGS BY GENDER}

\author{
SEBASTIÁN MORALES-NARANJO ${ }^{1}$ \\ VERÓNICA GARCÍA-OQUENDO ${ }^{2}$ \\ KARINA SILVA-JARAMILLO ${ }^{3}$
}

Recibido: 15 de mayo de 2017 Aceptado: 29 de agosto de 2017

\footnotetext{
${ }^{1}$ Pontificia Universidad del Ecuador, Facultad de Psicología, Quito, Ecuador (sebas-moralitos@hotmail.com).

2Pontificia Universidad del Ecuador, Facultad de Psicología, Quito, Ecuador (mvgarcia@puce.edu.ec).

${ }^{3}$ Pontificia Universidad del Ecuador, Facultad de Psicología, Quito, Ecuador (ksilva066@puce.edu.ec).
} 



\section{BURNOUT Y SATISFACCIÓN LABORAL: HALLAZGOS DIFERENCIALES POR GÉNERO}

\section{BURNOUT AND WORK SATISFACTION: DIFFERENTIAL FINDINGS BY GENDER}

Sebastián Morales-Naranjo, María Verónica García-Oquendo, Karina Alexandra Silva-Jaramillo

Palabras clave: Síndrome de Burnout, fatiga laboral, satisfacción laboral, género, organizaciones

Keywords: Burnout Syndrome, labor fatigue, labor satisfaction, gender, organizations

\section{RESUMEN}

En la actualidad, la sobrecarga laboral, el conflicto de roles entre la familia y el trabajo, la inestabilidad en la situación económica y política son factores que incrementan el esfuerzo que los trabajadores deben realizar para sostener adecuadamente su empleo. Esta energía demandada ha provocado que la fuerza trabajadora presente evidencias de experimentar el síndrome de Burnout o Síndrome de Estar Quemado, mismo que se relaciona con una disminución de la satisfacción laboral; sin embargo, no existen estudios en el Ecuador para determinar si el comportamiento de dichas variables se presenta en igual relevancia en hombres y mujeres. Por ello se aplicaron los cuestionarios Maslach Burnout Inventory, que evalúa el Síndrome de Burnout, y 
el S20/23 de satisfacción laboral a 163 hombres y 154 mujeres, se realizó un análisis descriptivo y posteriormente uno relacional entre ambas variables. Los resultados demuestran que en los hombres existe mayor agotamiento emocional y despersonalización; en cambio en las mujeres es la autoeficacia la dimensión con mayor afectación. Se concluye que no existen diferencias significativas entre géneros, probablemente por la tendencia a la equidad e igualdad que se pretende alcanzar en todos los aspectos de vida.

\section{ABSTRACT}

At present the overload labor, the conflict of roles between the family and the work, the economic and political instability situation, are factors that increase the effort workers must do to adequately keep their employment. This demanded energy caused Burnout Syndrome in the working force, which is related to a decrease in job satisfaction. However there are no studies in Ecuador to determine if the behavior of these variables is equal importance in men and women. That is the reason because the Maslach Burnout Inventory, which evaluates Burnout Sy- ndrome, and the $\$ 20 / 23$ of job satisfaction were applied to 163 men and 154 women. A descriptive and a relational analysis were performed between both variables. The results show that in men there is more emotional exhaustion and despersonalization while in women is self-efficacy the affected dimension. In conclusion there are no significant differences between genders, probably due to the tendency towards equity and equality that is intended to be achieved in all lifetime aspects.

\section{INTRODUCCIÓN}

El Síndrome de Burnout (SBO) está concebido como una afección de carácter psicológico con un componente altamente emocional y la sensación de agotamiento, en el cual el individuo no dispone de las herramientas personales básicas para afrontar las situaciones o demandas a las que se enfrenta (Gil Monte, 2001; Marrera y Grau 2005 citado en Ávila, Gómez y Montiel, 2010) y el tiempo que se encuentra expuesto a los estresores es prolongado, lo cual ocasiona malestar y 
presencia de síntomas específicos a nivel fisiológico, emocional, y conductual. (Maslach, Schaufeli y Leiter, 2001)

Por ser un fenómeno con alta afectación a nivel organizacional y personal es que en años recientes las investigaciones relativas al SBO alrededor del mundo han aumentado a más de 3000; en estos estudios se han abordado y profundizado los modelos teóricos, mecanismos de medición, causas y consecuencias (Pérez, 2012, Laba, Bosman y Buitendach, 2005 citado Gea-Izquierdo, García-Oquendo y Sáenz-Tinoco, 2015). Sin embargo, la mayoría de ellos se han enfocado en el campo de la salud, en profesionales asistenciales; actualmente, ya existen innumerables estudios en docentes y estudiantes (Aldrete, Preciado, Franco, Pérez y Aranda, 2008; Latorre y Sáez, 2009, Carlotto y Goncalves, 2008) y en menor escala en trabajadores no asistenciales y profesionales en general, trabajadores en organizaciones públicas y privadas, (García y Herrero, 2008, Gea-Izquierdo, García-Oquendo y Sáenz-Tinoco, 2015) pese a que conlleva a comportamientos preocupantes y relevantes como son el absentismo, poco compromiso institucional, disminución en el desempeño, alta rotación, conflictos en las relaciones y problemas de salud.

Así como la incidencia del SBO está en función del contexto profesional, campo ocupacional y antigüedad en el cargo (Bidlan, J.S. y Anupama, S., 2014), existen otras variables personales que afectan la prevalencia de su manifestación: los rasgos de personalidad, como la autoconfianza, autoestima y autoeficacia que son elementos que permiten un mejor afrontamiento y por ende disminuyen la sensibilidad para presentar este Síndrome (Chacón y Grau, 2004; Olmedo, 1997 citado en Ávila, Gómez y Montiel, 2010); el conflicto entre los roles asumidos por el trabajo y la familia (Yang, Liu, Liu, Zhang y Duan, 2017) y las características demográficas (edad, sexo, estado civil). (Ávila, Gómez y Montiel, 2010; García y Herrero, 2008; Contreras, Juárez y Murrain, 2008)

Los hallazgos encontrados en correspondencia con el género demuestran que la presencia del Síndrome se da especialmente en el género femenino de profesiones relacionadas con salud (enfermeros, médicos), presentando mayor intensidad en la subescala de agotamiento emocional frente a la despersonalización y a la realización personal. (Ávila, Gómez y Montiel, 2010). Esta tendencia de mayor incidencia del SBO en mujeres también se presenta en los siguientes grupos de ocupaciones: abogados, físicos, profesores, ministros de iglesia, conductores de autobús, trabajadores en publicidad y trabajadores en tecnologías de información, donde entre las dos dimensiones (agotamiento y compromiso) mayor afec- 
tación se observa en el agotamiento; sin embargo, las diferencias en cuanto al compromiso se dan según el campo ocupacional, de tal forma que las mujeres en profesiones de física, enfermería y profesoras presentan menor impacto en el compromiso que sus contrapartes masculinas; mientras que en conductores de autobús y ministras de iglesia sí existe mayor presencia de falta de compromiso en el género femenino que en el masculino; por último, en las otras profesiones no se ve ninguna diferencia debido a esta variable, situación que se replica en los oficiales de policía y veterinarios. (Falkum y Gjerlow, 2011, Bidlan, J.S. y Anupama, S., 2014, McCarty, M., Solomon, J. y Garland, B., 2007, Mastenbroek, Jaarsma, Demerouti, Muijtjens, Sherpbier, y Beukelen, 2014).

Según Onuoha y Akintola (2016), tampoco se encuentra evidencia de variaciones de la presencia de SBO en estudiantes universitarios por género; si bien es cierto, las mujeres presentaron un ligero incremento en la escala de agotamiento con respecto a los varones, este no fue estadísticamente significativo. Y en el caso de la sub-escala despersonalización o cinismo no hubo ninguna diferencia, pese a que en otros estudios mencionan que los hombres tienden a presentar puntajes mayores en la misma. (Purvanova y Muros, 2010)

Es en esta diversidad de hallazgos, con resultados totalmente opuestos, que se observa la necesidad de profundizar en estudios para determinar la situación en el Ecuador, ya que aún existen pocas investigaciones que contemplen la determinación del Síndrome de Burnout y la satisfacción laboral relacionadas con el género en la población trabajadora. Es relevante mencionar que la mayoría de investigaciones de esta índole se han producido en países como China, México, España e India; seguramente, las diferencias culturales en Latinoamérica pueden ser factores que inciden en la obtención de resultados novedosos.

Es en este espacio que encuentra fundamento esta investigación cuyo objetivo principal es analizar comparativamente por género la presencia de Burnout y satisfacción laboral en la fuerza trabajadora, así como determinar la relación entre estas variables en hombres y mujeres, aplicando los instrumentos Maslach Burnout Inventory (MBI) para el primer caso y el \$20/23 para el segundo, de tal forma que se pueda contribuir al planteamiento de intervenciones en riesgos psicosociales. 


\section{MATERIALES Y MÉTODOS}

Para el proceso investigativo se utilizó el estudio correlacional asociativo ya que se describe la relación entre dos variables consideradas en la investigación en un momento determinado. El SBO es la variable independiente e incluye tres dimensiones: agotamiento emocional, despersonalización, autoeficacia; y la variable dependiente es la satisfacción laboral entendida como el conjunto de actitudes generales del trabajador hacia sus actividades laborales (Robbins, 1998).

Dentro de la investigación se tomó en cuenta a la población de una empresa pública metropolitana dedicada a la distribución del agua, la cual cuenta con 1800 personas $(\mathrm{N}=1800)$. El procedimiento empleado es el método probabilístico de muestreo aleatorio simple (MAS), (Cochran, 1980). Con la fórmula correspondiente se obtuvo un total de 317 personas para el respectivo estudio $(n=317)$, divididos en 163 hombres y 154 mujeres, con edades que fluctúan entre los 22 y 70 años y que se encuentran en las Gerencias de Talento Humano, Operaciones, Comercial, Financiera, Jurídica, Logística, Ambiental, Técnica de Infraestructura y General.

En cuanto a los instrumentos, se utilizaron en primer lugar el cuestionario de Síndrome de Burnout (Maslach Burn- out Inventory) y el de satisfacción laboral (\$20/23), que fue creado por Maslach y Jackson (1981), en EEUU, y su propósito es medir los niveles de este síndrome tomando en cuenta las dimensiones de agotamiento emocional, despersonalización y autoeficacia. La investigación realizada por Manso (2006) muestra un alfa de validez con los siguientes puntajes para las dimensiones de este cuestionario: agotamiento emocional (0.82), despersonalización (0.80) y autoeficacia (0.85). En segundo lugar se utilizó el cuestionario creado por Meliá \& Peiró (1998), el cual consta de 23 items enfocados únicamente en satisfacción laboral, con escala de siete opciones de respuesta. Posee una nivel de confiabilidad de 0.92, idóneo para esta investigación y mide 5 factores: satisfacción con la supervisión, el ambiente físico, las prestaciones recibidas, satisfacción intrínseca del trabajo y con la participación.

Previo a la aplicación se socializó el procedimiento mediante el envío de un correo electrónico donde se señalaba la fecha y hora exacta de la aplicación a cada uno de los participantes de la muestra. Debido a que la muestra era muy numerosa, el proceso tardó aproximadamente una semana. El levantamiento de información se realizó por departamentos según el cronograma 
previamente establecido y el tiempo aproximado fue de 10 minutos.

Finalmente, se procedió a separar los cuestionarios según el género para su análisis y comparación individual, la tabulación se realizó en SPSS (versión 19); en primera instancia se efectuó un análisis descriptivo por cada variable y género, luego se comprobó la relación entre SBO y satisfacción laboral mediante la utilización del coeficiente de Pearson. Según Fernández (2001), el coeficiente de Pearson ( $r$ ) es el más utilizado para conocer la relación entre dos variables cuantitativas.

\section{RESULTADOS}

Atendiendo a los resultados obtenidos de la aplicación de los cuestionarios MBI y S20/23 a la muestra en general (154 mujeres y 163 hombres), los datos en la Tabla 1 arrojan que en la dimensión de agotamiento emocional del SBO el $5.68 \%$ de la muestra posee un nivel alto, $27.76 \%$ un nivel medio y un $66.56 \%$ posee un nivel bajo de padecimiento. En este caso, el agotamiento emocional es bajo, lo cual indica que no existe mayor impacto en características actitudinales tales como desmotivación, cansancio mental, aburrimiento, etc. (Miravalles, 2012). En cuanto a despersonalización, un $4.73 \%$ de los encuestados posee un nivel alto, un $19.56 \%$ un nivel medio y un $75.71 \%$ un nivel bajo de padecimiento del síndrome. Al igual que en la variable de agotamiento emocional existe mayor porcentaje en niveles bajos lo cual prueba que existen muy pocos empleados que se ven afectados en aspectos internos tales como percepción distorsionada de su cuerpo, automatismo, sentimientos de fantasía, insensibilidad a las personas, etc. (Simeon, 2016). Finalmente, en la dimensión de autoeficacia un $44.79 \%$ de la muestra posee un nivel alto, $29.34 \%$ un nivel medio y un $25.87 \%$ un nivel bajo. Cabe recalcar que en esta dimensión mientras más baja sea la puntuación existe mayor riesgo de padecer SBO. En esta variable, los resultados demuestran que menos de la mitad de los empleados sienten que su autoeficacia es óptima. Baja autoestima, sentimientos de "vacío" en cuanto a logros, evaluación negativa a las actividades realizadas son algunas de las características que determinan el aparecimiento del Síndrome (Miravalles, 2012). 
Tabla 1. Resultados de Síndrome de Burnout (Maslach Burnout Inventory)

\begin{tabular}{llcc}
\hline & & Número de personas evaluadas & Porcentaje (\%) \\
\hline Agotamiento Emocional & Alto & 18 & $5,68 \%$ \\
\cline { 2 - 4 } & Medio & 88 & $27,76 \%$ \\
\cline { 2 - 4 } & Bajo & 211 & $66,56 \%$ \\
\cline { 2 - 4 } & Total & 317 & $100 \%$ \\
\hline Despersonalización & Alto & 15 & $4,73 \%$ \\
\cline { 2 - 4 } & Medio & 62 & $19,56 \%$ \\
\cline { 2 - 4 } & Bajo & 240 & $75,71 \%$ \\
\cline { 2 - 4 } & Total & 317 & $100 \%$ \\
\hline Autoeficacia & Alto & 142 & $29.79 \%$ \\
\cline { 2 - 4 } & Medio & 93 & $100 \%$ \\
\cline { 2 - 4 } & Bajo & 317 & $25.87 \%$ \\
\cline { 2 - 4 } & Total & 32 & $234 \%$ \\
\hline
\end{tabular}

En cuanto a satisfacción laboral, en la Tabla 2, se tienen datos generales en donde únicamente el $1.58 \%$ de toda la muestra encuestada se encuentra insatisfecha, resaltando puntuaciones altas en insatisfacción por las prestaciones recibidas y la participación. La mayoría de empleados encuestados se muestra medianamente satisfechos dando como resultado el $86.75 \%$ del total de participantes, y finalmente están las personas satisfechas con un $11.67 \%$ en donde la variable de satisfacción intrínseca predomina como factor positivo para lograr este resultado. 
Tabla 2. Resultados de satisfacción laboral (S20/23)

\begin{tabular}{|c|c|c|c|c|c|c|c|c|}
\hline & $\begin{array}{c}\text { Insatisfecho } \\
(0-2)\end{array}$ & $(\%)$ & $\begin{array}{c}\text { Medianamente } \\
\text { Satisfecho } \\
(3-4-5)\end{array}$ & (\%) & $\begin{array}{c}\text { Satisfecho } \\
(6-7)\end{array}$ & $(\%)$ & $\begin{array}{l}\text { Total de } \\
\text { personas } \\
\text { evaluadas }\end{array}$ & Porcentaje \\
\hline $\begin{array}{l}\text { Satisfacción con } \\
\text { la supervisión }\end{array}$ & 6 & $1,89 \%$ & 254 & $80,13 \%$ & 57 & $17,98 \%$ & 317 & $100 \%$ \\
\hline $\begin{array}{l}\text { Satisfacción con } \\
\text { el ambiente físico }\end{array}$ & 11 & $3,47 \%$ & 240 & $75,71 \%$ & 66 & $20,82 \%$ & 317 & $100 \%$ \\
\hline $\begin{array}{l}\text { Satisfacción con } \\
\text { las prestaciones } \\
\text { recibidas }\end{array}$ & 16 & $5,05 \%$ & 271 & $85,49 \%$ & 30 & $9,46 \%$ & 317 & $100 \%$ \\
\hline $\begin{array}{l}\text { Satisfacción } \\
\text { intrínseca del } \\
\text { trabajo }\end{array}$ & 6 & $1,89 \%$ & 208 & $65,62 \%$ & 103 & $32,49 \%$ & 317 & $100 \%$ \\
\hline $\begin{array}{l}\text { Satisfacción con } \\
\text { la participación }\end{array}$ & 18 & $5,68 \%$ & 230 & $72,56 \%$ & 69 & $21,77 \%$ & 317 & $100 \%$ \\
\hline $\begin{array}{l}\text { Satisfacción } \\
\text { general }\end{array}$ & 5 & $1,58 \%$ & 275 & $86,75 \%$ & 37 & $11,67 \%$ & 317 & $100 \%$ \\
\hline
\end{tabular}

Por otro lado, es conveniente datos se encuentran en las Tablas 3 y 4. analizar los resultados por género, estos 
Tabla 3. Resultados de síndrome de quemado laboral en hombres (Maslach Burnout Inventory)

\begin{tabular}{llcc}
\hline & & Número de personas evaluadas & Porcentaje (\%) \\
\hline \multirow{4}{*}{ Agotamiento Emocional } & \multicolumn{1}{c}{ Alto } & 9 & $5,52 \%$ \\
\cline { 2 - 4 } & Medio & 53 & $32,52 \%$ \\
\cline { 2 - 4 } & Bajo & 101 & $61,96 \%$ \\
\cline { 2 - 4 } & Total & 163 & $100 \%$ \\
\hline \multirow{3}{*}{ Despersonalización } & Alto & 12 & $7,36 \%$ \\
\cline { 2 - 4 } & Medio & 43 & $26,38 \%$ \\
\cline { 2 - 4 } & Bajo & 108 & $66,26 \%$ \\
\hline & Total & 163 & $100 \%$ \\
\hline \multirow{3}{*}{ Autoeficacia } & Alto & 69 & $32,33 \%$ \\
\cline { 2 - 4 } & Medio & 49 & $30,06 \%$ \\
\cline { 2 - 4 } & Bajo & 45 & $27,61 \%$ \\
\cline { 2 - 4 } & Total & 163 & $100 \%$ \\
\hline
\end{tabular}

En la Tabla 3 se pueden observar los resultados de la muestra obtenidos en el SBO para sus tres dimensiones. En cuanto a agotamiento emocional, la mayor parte de los participantes presenta un porcentaje bajo (61.96\%), un número considerable de empleados presenta un nivel medio (32.52\%) y únicamente el $5.52 \%$ elevado. Sin embargo, es importante aclarar que de la muestra un $38.04 \%$ (5.52\% alto y $32.52 \%$ medio) percibe indicios de agotamiento emocional. En la dimensión de despersonalización, los resultados fueron similares a lo anteriormente descrito. Finalmente en la dimensión de autoeficacia se evidenció que menos de la mitad de los participantes (42.33\%) tiene un elevado nivel, un porcentaje considerable obtuvo resultados medios (30.06\%) y un porcentaje muy a la par de este, posee nivel bajo (27.61\%). Cabe destacar que calificaciones en estos rangos producen un decremento en la satisfacción laboral del empleado.

Como se puede ver, la autoeficacia es el factor que debe generar mayor preocupación ya que una cuarta parte de los trabajadores pertenecientes a la muestra percibe una sensación de no 
alcanzar sus objetivos y no acceder a oportunidades de crecimiento profesional dentro de la institución.

En cuanto a los resultados de satisfacción laboral expuestos en la Tabla 4 cabe recalcar que el mayor porcentaje de insatisfacción laboral está relacionado con la participación (6.75\%), esta hace referencia a las oportunidades que tiene el trabajador de ser considerado en la toma de decisiones dentro de la organización. Adicional, resalta un alto porcentaje de empleados insatisfechos (4.91\%) por la variable "prestaciones recibidas", aquí tiene mucho que ver la forma de negociación que tiene la institución con los empleados, los beneficios brindados y las oportunidades de crecimiento.

\section{Tabla 4. Resultados de Satisfacción Laboral en hombres (S20/23)}

\begin{tabular}{|c|c|c|c|c|c|c|c|c|}
\hline & $\begin{array}{c}\text { Insatisfecho } \\
(0-2)\end{array}$ & $(\%)$ & $\begin{array}{c}\text { Media- } \\
\text { namente } \\
\text { Satisfecho } \\
(3-4-5)\end{array}$ & $(\%)$ & $\begin{array}{c}\text { Satisfecho } \\
(6-7)\end{array}$ & $(\%)$ & $\begin{array}{l}\text { Total de } \\
\text { personas } \\
\text { evaluadas }\end{array}$ & Porcentaje \\
\hline $\begin{array}{l}\text { Satisfacción con la } \\
\text { supervisión }\end{array}$ & 3 & $1,84 \%$ & 128 & $78,53 \%$ & 32 & $19,63 \%$ & 163 & $100 \%$ \\
\hline $\begin{array}{l}\text { Satisfacción con el } \\
\text { ambiente físico }\end{array}$ & 7 & $4,29 \%$ & 120 & $73,62 \%$ & 36 & $22,09 \%$ & 163 & $100 \%$ \\
\hline $\begin{array}{l}\text { Satisfacción con } \\
\text { las prestaciones } \\
\text { recibidas }\end{array}$ & 8 & $4,91 \%$ & 134 & $82,21 \%$ & 21 & $12,88 \%$ & 163 & $100 \%$ \\
\hline $\begin{array}{l}\text { Satisfacción intrín- } \\
\text { seca del trabajo }\end{array}$ & 4 & $2,45 \%$ & 107 & $65,64 \%$ & 52 & $31,90 \%$ & 163 & $100 \%$ \\
\hline $\begin{array}{l}\text { Satisfacción con la } \\
\text { participación }\end{array}$ & 11 & $6,75 \%$ & 114 & $69,94 \%$ & 38 & $23,31 \%$ & 163 & $100 \%$ \\
\hline Satisfacción General & 3 & $1,84 \%$ & 139 & $85,28 \%$ & 21 & $12,88 \%$ & 163 & $100 \%$ \\
\hline
\end{tabular}

Por otra parte, existen trabaja- trínseca del trabajo", que tiene que ver dores muy satisfechos con algunas va- con que las actividades que realiza el riables, en primer lugar con el mayor trabajador estén acordes a su formación porcentaje (31.9\%) en "satisfacción in- y a su carrera; por ende, existe una co- 
modidad y una proactividad al realizar- ambiente físico" que hace referencia a la las. Además, existe un porcentaje ele- infraestructura del lugar de trabajo vado $(22.09 \%)$ con "satisfacción con el

Tabla 5. Resultados de síndrome de quemado laboral en mujeres (Maslach Burnout Inventory)

\begin{tabular}{clcc}
\hline & & Número de personas evaluadas & Porcentaje (\%) \\
\hline Agotamiento Emocional & Alto & 9 & $5,84 \%$ \\
\cline { 2 - 4 } & Medio & 35 & $22,73 \%$ \\
\cline { 2 - 4 } & Bajo & 110 & $71,43 \%$ \\
\cline { 2 - 4 } & Total & 154 & $100 \%$ \\
\hline Despersonalización & Alto & 3 & $1,95 \%$ \\
\cline { 2 - 4 } & Medio & 19 & $12,34 \%$ \\
\cline { 2 - 4 } & Bajo & 132 & $85,71 \%$ \\
\cline { 2 - 4 } & Total & 154 & $100 \%$ \\
\hline Autoeficacia & Alto & 72 & $36,75 \%$ \\
\cline { 2 - 4 } & Medio & 49 & $21,82 \%$ \\
\cline { 2 - 4 } & Bajo & 33 & $100 \%$ \\
\cline { 2 - 4 } & Total & 154 & \\
\hline
\end{tabular}

En la Tabla 5 se puede observar los datos de $\mathrm{SBO}$ en sus tres dimensiones tomando en cuenta al género femenino. En cuanto a agotamiento emocional la mayor parte de la población presenta un porcentaje bajo (71.43\%), mientras que en la dimensión de despersonalización se encontraron resultados similares al agotamiento emocional. Por último, en la dimensión de autoeficacia la mayor parte de los participantes posee niveles elevados (46.75\%), un $31.82 \%$ obtuvo resultados medios y un porcentaje menor obtuvo niveles bajos (21.43\%), valor que debe ser tomado en cuenta; pues, a largo plazo puede impactar en el nivel de satisfacción laboral. 
Tabla 6. Resultados de Satisfacción Laboral en mujeres (S20/23)

\begin{tabular}{|c|c|c|c|c|c|c|c|c|}
\hline & $\begin{array}{c}\text { Insatisfecho } \\
(0-2)\end{array}$ & $(\%)$ & $\begin{array}{c}\text { Medianamente } \\
\text { Satisfecho } \\
(3-4-5)\end{array}$ & $(\%)$ & $\begin{array}{c}\text { Satisfecho } \\
(6-7)\end{array}$ & (\%) & $\begin{array}{l}\text { Total de } \\
\text { personas } \\
\text { evaluadas }\end{array}$ & Porcentaje \\
\hline $\begin{array}{l}\text { Satisfacción con } \\
\text { la supervisión }\end{array}$ & 3 & $1,95 \%$ & 126 & $81,82 \%$ & 25 & $16,23 \%$ & 154 & $100 \%$ \\
\hline $\begin{array}{l}\text { Satisfacción con } \\
\text { el ambiente } \\
\text { físico }\end{array}$ & 4 & $2,60 \%$ & 120 & $77,92 \%$ & 30 & $19,48 \%$ & 154 & $100 \%$ \\
\hline $\begin{array}{l}\text { Satisfacción con } \\
\text { las prestaciones } \\
\text { recibidas }\end{array}$ & 8 & $5,19 \%$ & 137 & $88,96 \%$ & 9 & $5,84 \%$ & 154 & $100 \%$ \\
\hline $\begin{array}{l}\text { Satisfacción } \\
\text { intrínseca del } \\
\text { trabajo }\end{array}$ & 2 & $1,30 \%$ & 102 & $66,23 \%$ & 50 & $32,47 \%$ & 154 & $100 \%$ \\
\hline $\begin{array}{l}\text { Satisfacción con } \\
\text { la participación }\end{array}$ & 7 & $4,55 \%$ & 116 & $75,32 \%$ & 31 & $20,13 \%$ & 154 & $100 \%$ \\
\hline $\begin{array}{l}\text { Satisfacción } \\
\text { General }\end{array}$ & 3 & $1,95 \%$ & 135 & $87,66 \%$ & 16 & $10,39 \%$ & 154 & $100 \%$ \\
\hline
\end{tabular}

En la tabla anterior (Tabla 6) es importante destacar los resultados más significativos. En las mujeres se presenta mayor porcentaje de insatisfacción tomando en cuenta la variable "satisfacción con las prestaciones recibidas", aquí tiene mucho que ver la forma de negociación que tiene la organización con los empleados, los beneficios brindados, las oportunidades de crecimiento, etc. Además, se nota un nivel significativo de insatisfacción con el factor "satisfacción por la participación", esta hace referencia a las oportunidades que tiene el trabajador de participar en la toma de decisiones dentro de la organización.

En cuanto a las variables que resaltan por su nivel de satisfacción están "satisfacción intrínseca del trabajo", relacionada con las actividades que realiza el trabajador de acuerdo a su formación y a su carrera, por ende existe una comodidad y una proactividad al realizarlas; y "satisfacción con el ambiente físico" que 
hace referencia a la infraestructura del lugar de trabajo. Estos resultados fueron muy similares a los arrojados por el género masculino, con pequeñas variaciones que se discutirán posteriormente.
Por otro lado, para determinar la relación entre las variables por género se utilizó el coeficiente de Pearson y los resultados en el género masculino se muestran en la Tabla 7.

Tabla 7. Correlación entre Agotamiento Emocional, Despersonalización y Autoeficacia con Satisfacción Laboral (Hombres)

\begin{tabular}{|c|c|c|}
\hline & & Satisfacción General \\
\hline \multirow{3}{*}{ Agotamiento Emocional } & Correlación de Pearson &,- 467 \\
\hline & Sig. (bilateral) &, 000 \\
\hline & N & 163 \\
\hline \multirow{3}{*}{ Despersonalización } & Correlación de Pearson &,- 410 \\
\hline & Sig. (bilateral) &, 000 \\
\hline & N & 163 \\
\hline \multirow{3}{*}{ Autoeficacia } & Correlación de Pearson & ,302 \\
\hline & Sig. (bilateral) &, 000 \\
\hline & N & 163 \\
\hline
\end{tabular}

Como se puede observar el coeficiente de correlación de Pearson arroja un valor de $r=-0.467$, lo cual señala que existe una correlación negativa moderada entre SBO y satisfacción laboral.

Para la dimensión de despersonalización el coeficiente de correlación de Pearson arroja un valor de $r=-0.410$, lo cual señala que existe una correlación negativa moderada, por lo tanto a mayor nivel de despersonalización menor será la satisfacción.
En cuanto a la dimensión de autoeficacia en el género masculino es importante recordar que mientras más bajos sean los resultados existe una mayor posibilidad de insatisfacción laboral. Como se puede observar en la tabla de análisis anterior, el coeficiente de correlación de Pearson arroja un valor de r= 0.302 lo cual señala que existe una correlación positiva media. 


\section{Tabla 8}

Correlación entre Agotamiento Emocional, Despersonalización y Autoeficacia con Satisfacción Laboral (Mujeres)

\begin{tabular}{|c|c|c|}
\hline & & Satisfacción General \\
\hline \multirow{3}{*}{ Agotamiento Emocional } & Correlación de Pearson &,- 310 \\
\hline & Sig. (bilateral) &, 000 \\
\hline & N & 154 \\
\hline \multirow{3}{*}{ Despersonalización } & Correlación de Pearson &,- 160 \\
\hline & Sig. (bilateral) &, 000 \\
\hline & N & 154 \\
\hline \multirow{3}{*}{ Autoeficacia } & Correlación de Pearson & ,206 \\
\hline & Sig. (bilateral) &, 000 \\
\hline & N & 154 \\
\hline
\end{tabular}

En la Tabla 8, el coeficiente de $r=-0.160$, lo cual señala que existe una correlación de Pearson arroja un valor de correlación negativa baja. $r=-0.310$, lo cual señala que existe una

Como se puede observar en la correlación negativa media, por lo tanto tabla de análisis anterior el coeficiente mayor nivel de agotamiento emocional de correlación de Pearson arroja un valor menor será la satisfacción. de $r=0.206$ lo cual señala que existe una

En cuanto a la dimensión de des- correlación positiva baja entre autoefipersonalización el coeficiente de correla- cacia y satisfacción laboral en el género ción de Pearson arroja un valor de femenino. 


\section{DISCUSIÓN}

Existe una serie de diferencias y semejanzas entre los resultados arrojados por los dos cuestionarios tomando en cuenta el género de los empleados; en cuanto al SBO, considerando la primera dimensión, agotamiento emocional, se puede observar que en ambos casos existe un porcentaje muy bajo de personas que padecen intensamente esta sensación de fatiga y cansancio; sin embargo, en el caso de las mujeres existe un porcentaje mayoritario con un nivel bajo, lo cual denota que en el género masculino predomina esta característica; este resultado refleja información opuesta a la encontrada por otros autores (Andrada, 2006), (Albanesa, 2009), no obstante, es posible que tenga su explicación en la responsabilidad que recae sobre el hombre en el aspecto económico y manutención del hogar, ya que en nuestra sociedad aún se visualiza este comportamiento (Galvez, 2001). Otro factor que puede explicar estos resultados es que los hombres concentran su percepción de bienestar en los temas relativos al trabajo como esfera fundamental en la vida; actualmente, el país se encuentra en una época de crisis y recesión donde el desempleo y la posibilidad de ser despedido se ha intensificado en el último año, lo que incrementa la preocupación en las personas por lograr mantener su empleo. (Hoyo, 2010) Además, las mujeres culturalmente han desempeñado varios roles al mismo tiempo lo que ha permitido que se desenvuelvan con mayor facilidad en situaciones que exigen versatilidad.

En cuanto a la despersonalización se ve una diferencia más marcada: en el caso de los hombres existe un mayor porcentaje de empleados con niveles altos y medios lo cual hace referencia que son más propensos a padecer toda la sintomatología que engloba esta dimensión, como son ideas fantasiosas, automatismo, falta de iniciativa para crear relaciones interpersonales. Esto refuerza lo mencionado por Cornejo (2012), pues probablemente lo hacen como un mecanismo de defensa para sostener la situación en la que se encuentran dentro de la organización cuando tienen poco control y participación en la misma.

Finalmente, tomando en cuenta la autoeficacia, se puede resaltar que las mujeres poseen niveles más elevados; esto indica que sus expectativas y objetivos laborales han tenido mayor realización, la diferencia entre géneros en esta dimensión no es muy marcada pero sí tiene predominio por parte del grupo femenino. Probablemente se deba a que sus objetivos no están únicamente centrados en el trabajo sino que combinan 
otros roles (familiares y personales) que bien podrían provocar una percepción de logro de resultados, aunque el manejo de roles diversos pueden provocar afección en la dimensión de agotamiento emocional tal como lo explica en su estudio Díaz (2009), seguramente se han desarrollado destrezas en la capacidad de planificación y organización que permiten afrontar adecuadamente la realización de varias actividades a la vez y por ello no se presentó ese comportamiento en este caso.

Con todos los datos arrojados y analizados se puede ver que los hombres son más propensos a sufrir SBO en este entorno. En la mayoría de casos, los resultados son parejos pero siempre los hombres poseen niveles más altos en cuanto a las dos primeras dimensiones (agotamiento emocional y despersonalización) y más bajos en la tercera dimensión (eficacia).

Este último aspecto es de relevancia para la organización pues es donde tanto mujeres como hombres se concentran en un bajo nivel. Esto se puede explicar cuando no existe definición de objetivos en forma conjunta con el jefe inmediato, así Zarzar (1994) menciona en su estudio la importancia de participar y definir los objetivos en forma conjunta; por otro lado, también existen problemas con el estilo de liderazgo que imposibilita alcanzar las metas persona- les y organizacionales o no se han establecido planes de sucesión y de carrera que potencien las competencias a través de un desarrollo profesional (Soto, 2017).

Estos datos pueden también reflejarse en los resultados obtenidos en cuanto a satisfacción laboral, donde en ambos géneros existen pocas diferencias significativas en las variables que les causan insatisfacción y satisfacción. Se desprende que de los aspectos evaluados, los hombres en comparación con las mujeres están más insatisfechos en tres factores: ambiente físico, satisfacción intrínseca con el trabajo, satisfacción con la participación, mientras que el género femenino presenta mayor insatisfacción en dos aspectos: con la supervisión, lo que llama la atención pues según Robbins (1998) las mujeres suelen presentar mayor satisfacción con la autoridad, e insatisfacción con las prestaciones, pues lamentablemente en el género femenino aún no se ha logrado una igualdad salarial, fenómeno que está presente no solo en el Ecuador sino en diversos países del mundo (Castello, 2016). Cabe resaltar que en la satisfacción global o general las mujeres se encuentran más insatisfechas que los varones, pese a que únicamente en dos factores perciben de esta forma.

La mayoría de empleados insatisfechos se centran en el factor "prestaciones recibidas", siendo las mujeres 
un porcentaje alto, acontecimiento que puede explicarse al tomar en cuenta que en las empresas públicas el contrato colectivo es el beneficio más importante y por lo general ampara a los hombres ya que está dirigido a personas que trabajan en campo y que en su mayoría son de género masculino. Adicionalmente, existe insatisfacción en ambos géneros en el factor "satisfacción con la participación": los hombres presentan un mayor nivel de insatisfacción ya que se manifiesta en sus respuestas que no están siendo tomados en cuenta para los procesos y proyectos dentro de la organización, las mujeres también presentan este inconveniente pero el porcentaje es ligeramente menor. Esto se puede deber a que la participación de las mujeres en la parte familiar les sirva de catarsis a los sentimientos de malestar por no ser escuchadas, también es probable que las mujeres logren conversar de sus emociones lo cual baja el nivel de insatisfacción tal como lo afirma el artículo publicado por López (2016).

Por otra parte, los porcentajes mayoritarios en cuanto a satisfacción están ligados con la "satisfacción intrínseca del trabajo" y "satisfacción por el ambiente físico" en ambos géneros. Para el primer caso y según el manual del cuestionario los empleados están realizando tareas que les genera satisfacción, que están relacionados con su formación y su profesión, y si bien las mujeres muestran mayor satisfacción, la diferencia es mínima con respecto a los hombres. Finalmente, en el segundo factor que está relacionado con la infraestructura física se puede observar una ligera diferencia a favor de los hombres, esta satisfacción en ambos géneros está relacionada con las remodelaciones que se realizaron en la organización, recientemente fueron construidas nuevas instalaciones para todo el personal administrativo y operativo lo cual ha hecho que se cuente con equipos de tecnología avanzada y oficinas con todas las comodidades ergonómicas del caso.

En ambos casos (hombres y mujeres) las correlaciones arrojadas por el coeficiente de Pearson fueron similares, existen correlaciones negativas o inversas con las dos primeras dimensiones de SBO (agotamiento y despersonalización) y satisfacción laboral, esto señala que a mayor nivel en agotamiento emocional y despersonalización menor será la satisfacción en el empleado. En cuanto a la tercera dimensión de SBO y satisfacción laboral se encontró una correlación positiva o directa, lo que significa que a mayor nivel de autoeficacia mayor será la satisfacción laboral, tomando en cuenta que en esta dimensión siempre será mejor que los resultados sean altos.

Por esta razón es fundamental preocuparse por intentar disminuir el 
agotamiento y la despersonalización, con el fin de tener empleados más satisfechos, considerando que mientras tengan esta actitud positiva existirá menor rotación (Amorós, 2008), mayor compromiso institucional y ciudadanía organizacional (Betanzos, 2006).

\section{CONCLUSIONES}

El síndrome de quemado laboral es un tipo de riesgo psicosocial que se caracteriza por la excesiva carga laboral y por los pocos recursos para afrontarla, y que dependiendo de las condiciones bajo las cuales esté expuesto el trabajador (estrés, comportamiento de las personas que lo rodean en la organización, oportunidades de crecimiento, etc.) se irá desarrollando. Se comprueba que en la satisfacción laboral las brechas por género son leves.

En cuanto a los resultados obtenidos en cada género, cabe mencionar que no hay diferencias profundas; sin embargo, en los hombres se presenta mayor porcentaje en la dimensión de despersonalización, pues se debe considerar como punto prioritario el tema de autoeficacia, con mayor afectación en los hombres y que tomando en cuenta lo investigado por Herzberg, McClellan y Maslow con respecto a la importancia de cumplir y alcanzar los resultados, se conoce que los objetivos conllevan a la realización de metas profesionales. Los resultados de satisfacción laboral demuestran que existe disconformidad en ambos géneros con respecto a "satisfacción prestaciones recibidas" por la organización, "satisfacción en la toma de decisiones"; por esto, sería importante que la organización mejore la negociación en todos los temas legales así como la forma en que se da la selección y contratación del personal; aquí las más afectadas son las mujeres debido a la poca participación dentro del contrato colectivo firmado cada año. Por otra parte, y con una afectación ligeramente mayor en los hombres, la insatisfacción en la toma de decisiones podría ser mitigada con la inclusión de los empleados dentro de procesos, proyectos y objetivos que tenga la organización.

Se encontró una correlación significativa con respecto a las variables de síndrome de quemado laboral y satisfacción laboral: los resultados en ambos géneros fueron muy similares. En relación al agotamiento emocional y la satisfacción laboral se tiene una correlación negativa en hombres y mujeres; la relación entre despersonalización y satisfacción laboral nos indica una correlación negativa en 
hombres y mujeres, lo cual indica que a mayor nivel de despersonalización menor será la satisfacción en el empleado. Finalmente, la relación entre autoeficacia y satisfacción laboral fue una correlación positiva en ambos géneros.

Con la presente investigación, se determinó que en este tipo de organizaciones se cumple con la premisa de que las personas se sienten más satisfechas en un ambiente donde existen menores índices de síndrome de quemado laboral. Además, este estudio constituye un aporte para el mejoramiento continuo del ambiente laboral de la organización y una base teórica para futuros trabajos de investigación en el área de riesgo psicosocial en las organizaciones. Por último, las investigaciones que se hagan posteriormente deben romper las limitaciones que se presentaron en esta; especialmente, la muestra de estudio debe ser más específica con el fin de determinar características más particulares según la edad, personalidad, etnia, creencias, cargo, responsabilidades, etc. 


\section{BIBLIOGRAFÎA}

Albanesa, S. (2009). Burnout y Género. Revista de la Univerdad de San Luis, 7-9.

Aldrete, M. G., Preciado, M., Franco, S., Pérez, J.y Aranda, C. (2008). Factores psicosociales laborales y Síndrome de Burnout, diferencias entre hombres y mujeres docentes de secundaria, zona metropolitana de Guadalajara, México. Ciencia y Trabajo, 10(30), 138-142.

Amorós, E. (2008). Comportamiento Organizacional. Chiclayo: USAT.

Andrada, C. (2006). Diferencias por sexo, Síndrome de burnout y manifestaciones clínicas, en los médicos familiares de dos instituciones de salud, Guadalajara, México. Revista Costarricense de Salud Pública, 8.

Ávila, J., Gómez, L., Montiel, M., (2010). Características demográficas y laborales asociadas al Síndrome de Burnout en profesionales de la salud. Pensamiento Psicológico, 8 (15),39-52.

Betanzos, N. (2006). Compromiso organizacional en una muestra de trabajadores mexicanos. Revista de Psicología del Trabajo y de las Organizaciones, 11.

Bidlan, J.S. y Anupama, S., (2014). Occupational stress. Burnout, cop- ing and emotional intelligence: Exploring gender differences among different occupational groups of healthcare professionals. Indian Journal of Health and Wellbeing, 5(1), 149-154.

Carlotto, M. y Gonçalves, S. (2008). Predictores de Síndrome de Burnout en estudiantes universitarios. Pensamiento Psicológico, 4(10),101-109.

Castello, V. (2016). Diferencias salariales entre hombres y mujeres. Cinco Dias.

Cochran, W. (1980). Técnicas de Muestreo. México D.F.: CECSA.

Contreras, F., Juárez, F. y Murrain, E. (2008). Influencia del Burnout, la calidad de vida y los factores socioeconómicos en las estrategias de afrontamiento utilizadas por los profesionales y auxiliares de enfermería. Pensamiento Psicológico, 4(11), 29-44.

Cornejo, M. (2012). Redes sociales y relaciones interpersonales. Fundamentos en Humanidades, 23.

Díaz, G. (2009). Mujeres, trabajo y familia: una perspectiva de género desde América. Revista ITESO, 11-13.

Falkum, E., Gjerlow, O., (2011). Exploring within- and between-gender differences in burnout: 8 different occupational groups. Int Arch Oc- 
cup Environ Health, 84,813-824.

Fernandez, P. (3 de marzo de 2001). Fistierra. Obtenido de https://www. fisterra.com/mbe/investiga/var_ cuantitativas/var_cuantitativas2. pdf

Galvez, T. (2001). Aspectos Económicos de la Equidad de Género. Revista de las Naciones Unidas, 42-43.

García, J. M. y Herrero, S. (2008). Variables sociodemográficas y Síndrome de Burnout en una muestra multiocupacional del Hospital Psiquiátrico Penitenciario de Sevilla. Apuntes de Psicología, 26(3), 459477.

Gea-Izquierdo, E., García Oquendo, M., y Sáenz-Tinoco, D. (2015). Síndrome de Burnout en el sector público en Ecuador: primera evidencia en el sector no asistencial. En S. Martínez Alcántara, G. Gómez Pérez, C. Sánchez López \& J. Sandoval Ocaña, Efectos psicosociales de la crisis laboral y la precarización del trabajo (1st ed., 297-309). México: Universidad Autónoma Metropolitana.

Hoyo, M. (2010). INSHT. Obtenido de http://www.insht.es/InshtWeb/ Contenidos/Documentacion/ FICHAS\%20DE\%20PUBLICACIONES/FONDO\%20HISTORICO/DOCUMENTOS\%20DIVULGATIVOS/ DocDivulgativos/Fichero\%20pdf/

\section{Estres\%20laboral.pdf}

Latorre, I. y Sáez, J. (2009). Análisis del Burnout en profesores no universitarios de la región de Murcia (España), en función del tipo de centro docente: Público versus concertado. Anales de Psicología, 25(1), 86-92.

López, E. (2016). El equilibrio laboral aporta felicidad familiar. El Tiempo, 15.

McCarty, M., Solomon, J., Garland, B., (2007). Occupational stress and burnout between male and female police officers: Are there any gender differences? Polician: An International Journal of Police Strategies \& Management. 30(4), 672-691.

Manso-Pinto, Juan F... (2006). Estructura factorial del maslach burnout inventory - version human services survey - en Chile. Interamerican Journal of Psychology, 40(1), 111-114. Recuperado el 03 de abril de 2017, de http://pepsic.bvsalud.org/scielo.php?script=sci_arttext\&pi$d=$ S0034-96902006000100012\& $\mid-$ ng $=$ pt\&tlng=es.

Maslach, C., \& Jackson, S. (1981). Maslach Burnout Inventory. Palo Alto: Consulting Psychologists Press.

Maslach.C., Schaufeli, B. y Leiter, M. P. (2001). Job Burnout. Annual Re- 
view Psychology, 52, 397- 422.

Mastenbroek, N., Jaarsma, A., Demerouti, E., Muijtjens, A., Sherpbier, A. y Beukelen, P., (2014). Burnout and engagement, and its predictors in young veterinary professionals: the influence of gender. Veterinary Record. 174, 144.

Meliá, J., \& Peiró, J. (1998). Psicología de la Seguridad. Obtenido de http:// www.uv.es/ meliajl/Research/ Cuest_Satisf/S20_23.PDF

Miravalles, J. (2012). Características del SBO. Obtenido de http://www. javiermiravalles.es/síndrome\%20 burnout/Caracteristicas.pdf

Onuoha, U. y Akintola, A., (2016). Gender Differences in Self-Reported Academic Burnout among Undergraduates. Gender\&Behavior, 14(1), 7110-7116.

Purvanova, R. y Muros, J., (2010). Gender differences in burnout: A meta-analysis. Journal of Vocational Behavior, 77 (2), 168-185.

Robbins, S. (1998). Fundamentos de comportamiento organizacional. México. D.F.: Prentice Hall.
Secretaría Nacional de Planificación y Desarrollo, (2013). Plan Nacional del Buen Vivir 2013-2017. Ecuador. Simeon, D. (2016). Manual MSD. Obtenido de http://www.msdmanuals.com/es-ec/hogar/ trastornos-de-la-salud-mental/ trastornos-disociativos/trastorno-de-despersonalizaci\%C3\%B3n-desrealizaci\%C3\%B3n

Soto, B. (2017). Qué es y cómo desarrollar un plan de carrera. Obtenido de http://www.gestion.org/recursos-humanos/30690/desarroIlar-plan-carrera/

Yang, S., Liu, D., Liu, H., Zhang, J., Duan, Z. (2007).Relationship of work-family conflict, self-reported social support and job satisfaction to burnout syndrome among medical workers in southwest China: A cross-sectional study. Plos One,12 (2).

Zarzar, C. (1994). LA DEFINICION DE OBJETIVOS DE APRENDIZAJE. Redalyc, 19. 